\title{
Privatisierung der Strafverfolgung - Königsweg oder Irrweg?
}

\author{
RAINER SIEG \\ Universität Passau
}

\section{Einführung}

2. Sachverhaltsaufklärung durch Privatermittler

2.1 Überprüfung von elektronischen und schriftlichen Geschäftsunterlagen

2.2 Interviews mit potenziellen Tätern und Zeugen

2.2.1 Wahrung der Beteiligungsrechte der Arbeitnehmervertretungen

2.2.2 „Einladung“ des Mitarbeiters zum „Interview“

2.2.3 Begleitpersonen für den Interviewten

2.2.4 Durchführung der Befragungen durch die externen privaten Ermittler

2.2.5 Problem: Befragung durch ausländische Rechtsanwälte

2.2.6 Einsichtsrecht in die Protokolle nach der Befragung?

2.2.7 Einsichtsrecht, da die Mitschriften Teil der Personalakte sind?

2.2.8 Aussageverpflichtung von Arbeitnehmern bei unternehmensinterner Befragung?

2.2.9 Verwertbarkeit von unternehmensinternen Aussagen in Strafverfahren?

2.3 Privatermittler als Hilfsorgan der Staatsanwaltschaft?

2.4 Privatermittler als Hilfsorgan ausländischer Behörden?

3. Unternehmensseitige Maßnahmen zur Aufklärung von Compliance-Verstößen

3.1 Amnestieangebot

3.2 Aufforderung zum Whistleblowen

4. Exkulpationspapier

5. Mindeststandards für unternehmensinterne Untersuchungen

6. Fazit: Privatisierung der Strafverfolgung nur vorübergehender Ausweg 
I. Der durch die Unternehmensleitung organisierte umfassende Einsatz von Privatpersonen zur systematischen Aufklärung von vermuteten Wirtschaftsstraftaten ist eine im US-amerikanischen Rechtskreis seit Jahrzehnten erprobte und anerkannte Methode der Sachverhaltsaufklärung. In Deutschland war diese Form unternehmensinterner Privatermittlungen statt oder neben der seit Jahrhunderten tradierten staatlichen Strafverfolgung unbekannt. Das änderte sich schlagartig mit der spektakulären Korruptionsaffäre bei Siemens in 2007. Die Tatsache und die besonderen Begleitumstände der Aufklärung von Korruptionsvergehen durch die amerikanische Anwaltskanzlei Debevoise \& Plimpton und deren Zusammenarbeit mit deutschen aber auch US-amerikanischen Behörden fand ein überaus starkes Medienecho (z. B.: Frankfurter Allgemeine vom 7.2.2007: „Die Superanwälte von Siemens“; ZEIT ONLINE vom 15.3.2008: „Prozess der Selbstreinigung - Mit Milliardenaufwand arbeitet Siemens Korruptionsfälle auf. Aber können konzerninterne Ermittlungen die Arbeit der Staatsanwälte ersetzen?“`)!

Dass Siemens kein Einzelfall geblieben ist, sondern es in den letzten Jahren „bei Fällen von internationaler Wirtschaftskriminalität längst üblich geworden ist, die Ermittlungsarbeit (die normalerweise Polizisten und Staatsanwälte machen) weitgehend an Anwaltskanzleien auszulagern" hat Marcus Rohwetter in einem Artikel über die Diesel-Affäre bei Volkswagen (DIE ZEIT Nr. 41/2015, vom 8.10.2015) aufgezeigt. „Die US-Justiz verspricht Unternehmen Strafmilderung, wenn sie auf diese Art kooperieren. Das bedeutet eine schleichende Privatisierung der Strafverfolgung." Private Ermittler, die auf das internationale Wirtschaftsstrafrecht spezialisiert sind, arbeiten schneller und effizienter als staatliche Ermittler, werden aber auch deutlich besser bezahlt als diese. Interne Ermittlungen (auch in Deutschland gelegentlich „Internal Investigations“ genannt) haben sich zu einem lukrativen Geschäftsmodell insbesondere für amerikanische Anwaltskanzleien entwickelt, denn nur diese werden von den amerikanischen Behörden akzeptiert. Manche sprechen deshalb auch von einem ,amerikanischen Rechtsimperialismus“ (DaimlerChrysler - „US-Imperialismus“ beklagt, www.focus.de vom 17.9.2006, abrufbar unter: http://www.focus.de/finanzen/news/daimlerchrysler_aid_115654. html, letzter Zugriff: 6.04.2017). Allein Siemens zahlte 204 Millionen $€$ an Honoraren für $D P$ und weitere 350 Millionen $€$ an die von den Anwälten benötigten Helfer bei der Wirtschaftsprüfungsgesellschaft Deloitte Touch Tohmatsu.

Im Folgenden soll untersucht werden, ob die Privatisierung der Strafverfolgung bei Wirtschaftsstrafsachen auch in Deutschland (s. Taschke, Zur Entwicklung der Verfolgung von Wirtschaftsstrafsachen in der Bundesrepublik Deutschland, Teil 3: Auf dem Weg zur Privatisierung der Strafverfolgung, NZWiSt 2012, 89; Momsen, Verlagerung der Strafverfolgung auf Private im Wirtschaftsrecht Chance oder Irrweg) sich als Königsweg oder als Irrweg erwiesen hat.

\footnotetext{
${ }^{1}$ http://www.zeit.de/2008/12/Selbstreinigung-Siemens (letzter Zugriff: 6.04.2017).
} 
II. Der Prüfungsausschuss des Aufsichtsrats der Siemens AG hatte 2007 die amerikanische Rechtsanwaltskanzlei Debevoise\& Plimpton (im Folgenden: DP) und diese die Wirtschaftsprüfungskanzlei Deloitte Touch Tohmatsu mit der weltweiten Durchführung einer unabhängigen Untersuchung gegebenenfalls vorliegender Compliance-Verstöße (ob und in welchem Umfang es zu ungerechtfertigten Zahlungen, Korruption, Bestechung, Geldwäsche oder vergleichbarem Fehlverhalten gekommen ist) sowie der internen Systeme zur Durchsetzung der Compliance beauftragt. Um eventuelle Compliance-Verstöße aufzudecken, sollte $D P$ Interviews führen und Geschäftsunterlagen des Unternehmens überprüfen. $D P$ sollte ausschließlich an das Compliance-Komitee des Aufsichtsrats berichten.

Angesichts der Vielzahl von Verstößen, die in einem weltweit tätigen Konzern vorkommen können, ist es selbstverständlich, dass der Vorstand nicht jedem einzelnen Rechtsverstoß durch Unternehmensangehörige selbst nachgehen muss. Es muss ihm gestattet sein, die Befugnisse zur Aufklärung von vermuteten Regelverstößen durch Auswertung von Beweismaterial und Befragung von Mitarbeitern auf externe Dritte mit einschlägiger weltweiter Erfahrung auszulagern/ outzusourcen (OLG München, Urteil vom 25.3.2009 - 7 U 4835/08; Mengel in: Knierim/Rübenstahl/Tsambikakis, Internal Investigations, Kap.13 Rn. 39; Wewerka, Internal Investigations, S. 127). Zu diesen Dritten gehören auch externe Rechtsanwälte (s. dazu Wewerka, Internal Investigations, S. 127; Wuttke, Straftäter im Betrieb, S. 213; OLG München, NZG 2009, 665, 666).

Mit einem Rundschreiben des Zentralvorstands der Siemens AG wurden (in Ausübung des Arbeitgeber-Direktionsrechts) alle Mitarbeiter des Konzerns verpflichtet, bei dieser Untersuchung mit den unternehmensexternen Privatermittlern vollumfänglich zu kooperieren. DP musste von allen Mitarbeitern Zugang sowohl zu schriftlichen als auch zu elektronischen Geschäftsunterlagen gewährt werden. DP konfiszierte keine Geschäftsunterlagen, war aber befugt, zur weiteren Auswertung Kopien von belastendem Material anzufertigen, wovon sie intensiv Gebrauch machte.

III. Stand eine Abteilung im Verdacht illegaler Handlungen, kam ein (nur) für die Datensicherung zuständiger Rechtsanwalt in das Büro und stellte nach Befragung der Büroangehörigen zunächst fest, welches elektronische Equipment vorhanden ist. Dieses wurde mitgenommen (gelegentlich kam es dabei zu Diskussionen, ob es sich bei dem Laptop um ein dienstliches oder privates Gerät handelt), die darin elektronisch gespeicherten Daten unmittelbar danach auf eigene Festplatten heruntergeladen und die Geräte (zur Aufrechterhaltung der Arbeitsfähigkeit der Arbeitnehmer) möglichst schnell wieder zurückgegeben. Danach wurden alle Akten und sonstigen schriftlichen Unterlagen gesichtet. Verdächtig erscheinende Schriftstücke wurden ebenfalls mitgenommen und kopiert. Allen Mitarbeitern war durch Firmenrichtlinie streng verboten, während der Untersuchungen Computer, 
Laptops oder sonstige IT-Ausrüstung zu vernichten oder nur aus dem Büro zu entfernen oder E-Mails zu löschen. Mit den beauftragten Kanzleien wurden Data Transfer Agreements abgeschlossen, die u.a. Vorgaben zur Etablierung eines angemessenen Datenschutzniveaus und (in Anlehnung an Regelungen zur Auftragsdatenverarbeitung) zu technischen und organisatorischen Maßnahmen zum Schutz personenbezogener Daten enthielten. Auf die Daten von Arbeitnehmervertretern, Datenschutzbeauftragten und Betriebsärzten durfte nicht zurückgegriffen werden. Für die Einhaltung des Datenschutzes durch die Mitarbeiter der Beraterkanzleien war die Datenschutzorganisation des Unternehmens zuständig.

Auf Grundlage der sichergestellten Geschäftsunterlagen wurden dann verdächtig gewordene Mitarbeiter zu Interviews eingeladen. Da die Interviewer sich durch die Materialauswertung auf die Befragung gut vorbereiten konnten, wurden die Befragten darauf hingewiesen, dass es keinen Sinn macht, die Existenz von belastenden Unterlagen zu leugnen.

IV. Zur Wahrung der Beteiligungsrechte der Arbeitnehmervertretung (allgemeines Informationsrecht des Betriebsrats nach $\S 80$ Abs. 2 Betriebsverfassungsgesetz und Mitbestimmung über Fragen der Ordnung des Betriebs und des Verhaltens der Arbeitnehmer im Betrieb nach $\S 87$ Abs. 1 Nr. 1 Betriebsverfassungsgesetz) wurde am 26.7.2007 mit dem Siemens-Konzernbetriebsrat eine entsprechende Konzernbetriebsvereinbarung „Compliance“ geschlossen.

Mit der gesetzlichen Vertretung der Leitenden Angestellten (ca. 2\% aller Mitarbeiter), dem Konzernsprecherausschuss, wurde keine Vereinbarung geschlossen, obwohl die allermeisten der betroffenen Mitarbeiter aus diesem Kreis stammen. Zwar hat der Konzernsprecherausschuss als Interessenvertretung der Leitenden Angestellten keinen Rechtsanspruch auf Abschluss einer Vereinbarung, eine freiwillige Vereinbarung wäre aber gemäß $\S 28$ Sprecherausschussgesetz möglich gewesen. So hat die Firmenseite mit der Ablehnung einer diesbezüglichen Bitte der Vertretung der Leitenden Angestellten eine Chance vertan, bei den im Wesentlichen betroffenen Führungskräften für Vertrauen auf ein rechtmäßiges und vor allem faires Verfahren zu werben. So konnte der Eindruck entstehen, dass die Firmenvertreter den einfacheren Weg gegangen sind, mit der Vertretung der nur marginal betroffenen Mitarbeitergruppen eine Vereinbarung über eine möglichst „konsequente“ Durchführung der Untersuchung zu schließen. Dies war auch taktisch klug, zumal durch das Fehlverhalten der (nicht vom Betriebsrat vertretenen) Vorgesetzten viele Arbeitsplätze insbesondere der „einfachen“ (vom Betriebsrat vertretenen) Arbeitnehmer gefährdet wurden, wofür letztere naturgemäß wenig Verständnis hatten.

V. Die „Einladung“ des Mitarbeiters zur „Interview“ genannten Befragung erfolgte durch eine zur logistischen Unterstützung der externen Berater eingerich- 
tete Firmenabteilung (Projekt Office Compliance Investigation). Diese informierte über Interview-Ort/Termin, Interviewer (inkl. Lebenslauf in Englisch), Durchführung des Interviews, Kontaktdaten des Zeugenbeistands und bat den Mitarbeiter um seinen Lebenslauf.

VI. Damit ein (in der Regel nicht juristisch vorgebildeter) Befragter nicht „schutzlos" der strukturellen Berufs-Überlegenheit von ermittelnden Rechtsanwälten ausgeliefert ist, konnte er als „Zeugenbeistand“ einen von der Firmenleitung bezahlten Rechtsanwalt der Kanzlei Baker\&McKenzie mitbringen. Diese Kanzlei stand den Befragten auch zur Vorbereitung auf das Interview und im Zusammenhang mit der Anforderung und Durchsicht von Geschäftsunterlagen durch $D P$ beratend zur Verfügung. In die Befragung der Mitarbeiter wurde sie durch $D P$ aber nicht eingebunden.

Der Arbeitnehmer konnte statt eines Vertreters dieser Kanzlei auch einen (von ihm selbst zu bezahlenden) Rechtsanwalt seines Vertrauens hinzuziehen.

Darüber hinaus konnte er auch einen nach deutschem Arbeitnehmervertretungsrecht zuständigen Betriebsrat oder als Leitender Angestellter ein Sprecherausschussmitglied seines Vertrauens um Begleitung bitten.

VII. Die Befragungen von Mitarbeitern erfolgten jeweils mindestens durch zwei Rechtsanwälte der Kanzlei, dauerten oft mehrere Stunden und wurden (in Deutschland) teilweise auf Englisch abgehalten. Während einer der Rechtsanwälte die Fragen stellte, wurden dessen Fragen und die Antworten des Befragten von dem anderen Rechtsanwalt (als einzige erkennbare Aufgabe) schriftlich in englischer Sprache auf einem PC bzw. Laptop festgehalten. Ein Personal-Luxus, der deutschen Staatsanwaltschaften versagt ist. Darüber hinaus dürften sich deutsche Staatsanwälte auch nur der Gerichtssprache, also des Deutschen, bedienen.

Der Ablauf der in den Geschäftsräumen des Unternehmens stattfindenden Befragungen gestaltete sich in der Regel wie folgt:

(1) Allgemein gehaltene Fragen nach dem beruflichen Werdegang, der Zusammenarbeit mit Kollegen, Zusammensetzung des Einkommens und Incentivierung.

(2) Annäherung an „kritische“ Themen durch Fragen u.a. nach dem dienstlichen Verhalten, Anbahnung von Geschäften, Geschäftspartnern und Geschäftspraktiken.

(3) Bei Bedarf Folgeinterviews zur Klärung spezifischer Sachverhalte.

Die Befragungen wurden von den Rechtsanwälten zwar euphemistisch „Interviews" genannt, einige Interviewte fühlten sich allerdings einer verhörsähnlichen Lage ausgesetzt (Maschmann, AuA 2009, 72, 76; Rudkowski, NZA 2011, 612; Wastl/Litzka/Pusch, NStZ 2009, 68, 71). Der prominenteste von DP Interviewte, der frühere Vorstandsvorsitzende und Aufsichtsratsvorsitzende der Siemens $A G$, 
Heinrich v. Pierer, schrieb in seiner Autobiografie „Gipfelstürme“: „Eigentlich hätte man sie Verhöre nennen sollen.“

Den Rechtsanwälten ist es rechtlich gestattet zum „Druckaufbau“ einen Hinweis auf die Möglichkeit der Anzeige unrechtmäßigen Verhaltens bei den staatlichen Behörden zu geben, auch wenn manche Befragten dies als Nötigung zur Aussage werten. Mancher Interviewte empfand diese Alternative angesichts der erlebten Befragung aber gar nicht mehr als so abschreckend. Spontane Äußerung eines interviewten Topmanagers nach Abschluss der Befragung: „Da hätte ich ja gleich zur Staatsanwaltschaft gehen können“ (manager magazin vom 20.4.2011). Das könnte für die Rechtsposition des Befragten sogar von Vorteil sein, denn die Staatsanwaltschaft hat nach $\S 160$ Abs. 2 Strafprozessordnung ,nicht nur die zur Belastung, sondern auch die zur Entlastung dienenden Umstände zu ermitteln“.

Dagegen, wird die besondere Atmosphäre der Interviews auch dadurch geprägt, dass die Ermittler keineswegs zur Objektivität verpflichtet und damit verpflichtet sind, Umstände zu ermitteln, die den Mitarbeiter entlasten, sondern vielmehr einseitig die Interessen des Unternehmens vertreten“ (Wewerka, Internal Investigations, S. 101; siehe auch: Wastl, WirtschaftsWoche, www.wiwo.de/unternehmen-maerkte/szenisch-effektvoll-und-ueberraschend-303167/; Knierim, StV 2009, 324, 325; Hassemer/Matussek, Opfer als Verfolger, S. 26 f., zuletzt aufgerufen: 6.04.2017). „Ziel der Ermittlungen ist die Entlastung des Unternehmens als Ganzem, die jedoch nur durch gezielte Belastung einzelner Mitarbeiter erfolgen kann. In diesem Sinne stehen alle Mitarbeiter zunächst unter Generalverdacht; der Unschuldsvermutung wird keinerlei Bedeutung beigemessen" (Wewerka, Internal Investigations, S. 149).

Anwendung von Zwang, Drohung, Nötigung und Täuschung ist nicht nur bei Befragungen durch Polizisten und Staatsanwälte, sondern auch in Interviews durch Private verboten, weil sie die Menschenwürde des Befragten verletzen würde und dieses Grundrecht aus Art. 1 Abs. 1 Grundgesetz auch unmittelbar zwischen Privaten anzuwenden ist. Aber auch ohne Grundrechtsverletzung ist es den Anwälten der Anwaltskanzlei DP (die sich nach Einschätzung des manager magazins ihren Ruf als gefürchtetste Kanzlei weltweit hart erarbeitet hat) gelungen, in der deutschen Wirtschaft „Angst und Schrecken“ zu verbreiten (manager magazin vom 20.4.2011), was der Aussagebereitschaft der Befragten zuträglich gewesen sein soll.

VIII. Staatsanwaltschaftliche Vernehmungen müssen grundsätzlich in der Gerichtssprache und nach dem Landesrecht durchgeführt werden. Das ist deshalb kein Problem, weil die Staatsanwälte eine juristische Ausbildung im jeweiligen Land erfolgreich absolviert haben müssen. Was aber, wenn die ermittelnden Rechtsanwälte aus dem Ausland kommen und weder die Landessprache noch das Landesrecht beherrschen? Um Mitarbeitern eine herkunftsbedingte sprachliche 
„Waffenungleichheit“ (selbst bei guten Englischkenntnissen) in heiklen Themen zu ersparen, wurden die Interviews bei Siemens in Deutschland mit Unterstützung von Simultandolmetschern in der Muttersprache der Befragten geführt. Jeder der schon einmal Verhandlungen in einem fremden Land mit Unterstützung von Dolmetschern geführt hat (ich kann mich noch gut an meine eigenen Erfahrungen in Polen erinnern), weiß, wie schwierig es sein kann, eine Aussage nicht nur wörtlich zu übersetzen, sondern deren gewollte Bedeutung in den anderen Sprachkulturkreis ohne Missverständnisse zu übertragen. Komplexes „Juristendeutsch“ ist nur schwer in ebenso komplexes „Juristenenglisch“ zu übersetzen. Diese Erfahrung musste ein (der englischen Sprache und des amerikanischen Rechts weitgehend mächtiger) Befragter machen, als er merkte, dass der Dolmetscher den Rechtsbegriff Untreue mit Unterschlagung übersetzte. Grund: Das amerikanische Rechtssystem kennt den deutschen Straftatbestand der „Untreue“ (§ 266 StGB) nicht. Einem anderen nicht ähnlich sprach- und rechtskundigem Interviewten, wäre dieser nicht unwesentliche Übersetzungsfehler gar nicht aufgefallen.

Um derartige kulturell bedingte Missverständnisse zu vermeiden, müssen ausländische Interviewer ein großes Interesse daran haben, dass die Befragten nach Ende des Interviews, die protokollierten Fragen und Antworten noch einmal in aller Ruhe auf Richtigkeit überprüfen. Schließlich sind sie es, die als landessprachunkundige Ausländer, etwas von einem Inländer wollen. Falls der Mitarbeiter sich des Englischen nicht so (rechts)sicher fühlt, müssen ihm seine Aussagen in seiner Muttersprache vorgelegt werden.

IX. Umso überraschender ist, dass ein selbstverständlich wirkendes Einsichtsrecht des Befragten von der amerikanischen Rechtsanwaltskanzlei DP strikt abgelehnt wurde.

Die Begründung des $D P$-Chefs Yannett hierfür: „Mitschriften von Interviews von $D P$ sind vertraulich und werden weder an das Unternehmen noch an die Öffentlichkeit gegeben. Nach US-Recht wäre jede Form der Kenntnisnahme durch den Interviewten eine Anerkennung des Inhalts mit entsprechenden rechtlichen Folgen.“

Inwieweit ausländisches Recht überhaupt in der Lage ist, die inländischen Rechte eines Arbeitnehmers gegen seinen Arbeitgeber außer Kraft zu setzen, ist äußerst zweifelhaft und bedürfte einer tieferen wissenschaftlichen Betrachtung, die den Rahmen dieses Beitrags sprengen würde.

Selbst wenn man den Arbeitnehmer aufgrund seiner Treuepflicht gegenüber seinem Arbeitgeber ( $§ 241$ Abs. 2 BGB) ausnahmsweise für verpflichtet hielte, bei der Ausübung von Rechten Rücksicht auf die Belange des Arbeitgebers zu nehmen, die sich aus der Befolgung ausländischer Rechtsvorschriften ergeben können, kann dies nicht soweit gehen, berechtigte Schutzbelange hintanzustellen, wenn sich dadurch die eigene Rechtsposition gravierend verschlechtert, ohne dass der Arbeitgeber hierfür einen für ihn wesentlichen Rechtsvorteil erlangt. 
Vielmehr ist der Arbeitgeber auch hier zur Einhaltung des deutschen Arbeitsrechts verpflichtet.

Das gilt umso mehr, als eine Verschlechterung der Arbeitgeberposition durch das Einsichtsrecht nicht ernsthaft zu befürchten ist, weil sich alle Vorgänge betriebsintern abspielen und der Arbeitnehmer kein Dritter ist, sondern ein an die Verschwiegenheitspflicht (§ 241 Abs. 2 BGB) gebundener Betriebsangehöriger.

Ganz im Gegenteil würde sich durch ein Einsichtsrecht die Rechtsposition des Arbeitgebers sogar noch verbessern, da damit Missverständnisse und Fehler bei der Protokollierung unverzüglich entdeckt und sofort korrigiert werden könnten.

Mitarbeitern, die sowohl von der im Auftrag des Arbeitgebers handelnden Rechtsanwaltskanzlei, als auch von der Staatsanwaltschaft zu (ggf. den gleichen) Compliance-Themen befragt wurden, fiel es besonders schwer, diese Verweigerungshaltung nachzuvollziehen. Denn bei der Staatsanwaltschaft wurden sie ausdrücklich dazu aufgefordert, das Vernehmungsprotokoll eingehend zu lesen, ggf. auszubessern und zu unterschreiben. Im Übrigen wurden gelegentlich auch Aussagen des Mitarbeiters bei der jeweils anderen Vernehmungsstelle hinterfragt. Da wäre es gut für den Befragten, zu wissen, was die jeweiligen Befrager über ihn notiert haben.

Die Aussage des US-Anwalts Yannett, ,nach US-Recht wäre jede Form der Kenntnisnahme eine Anerkenntnis des Inhalts" ist für einen nach kontinentaleuropäischem Recht sozialisierten Rechtsanwalt nur schwer nachvollziehbar. Bevor man anderer Meinung als ein Kollege sein kann, muss man doch erst einmal zur Kenntnis nehmen, welche Meinung dieser hat. Die ggf. gewollte Anerkennung einer Aussage erfolgt erst durch die Unterschrift des Befragten. Zu dieser kann aber ein Vernommener - selbst wenn er den Aussagetext ganz in seinem Sinne korrigieren durfte - noch nicht einmal von der Staatsanwaltschaft gezwungen werden.

Hintergrund der strikten Verweigerungshaltung ist wohl US-amerikanisches Zivilprozessrecht.

Federal Rules of civil procedure

Rule 26. Duty to Disclose; General Provisions Governing Discovery

(b) DISCOVERY SCOPE AND LIMITS.

(3) Trial Preparation: Materials.

(A) Documents and Tangible Things. Ordinarily, a party may not discover documents and tangible things that are prepared in anticipation of litigation or for trial by or for another party or its representative (including the other party's attorney, consultant, surety, indemnitor, insurer, or agent). But, subject to Rule 26(b) (4), those materials may be discovered if:

(i) they are otherwise discoverable under Rule 26(b)(1); and

(ii) the party shows that it has substantial need for the materials to prepare its case and cannot, without undue hardship, obtain their substantial equivalent by other means. 
(B) Protection Against Disclosure. If the court orders discovery of those materials, it must protect against disclosure of the mental impressions, conclusions, opinions, or concerning the litigation.

(B) Schutz gegen Offenlegung. Wenn das Gericht die Offenlegung dieser Materialien anordnet, muss es vor Offenlegung von mentalen Eindrücken, Schlussfolgerungen oder den Rechtsstreit betreffenden Meinungen schützen.

(C) Previous Statement. Any party or other person may, on request and without the required showing, obtain the person's own previous statement about the action or its subject matter. If the request is refused, the person may move for a court order, and Rule 37(a)(5) applies to the award of expenses.

(C) Frühere Erklärung. Jede Partei oder jede andere Person kann auf Antrag und ohne die erforderliche Anzeige die frühere Erklärung der Person über die Aktion oder ihren Gegenstand erhalten.

A previous statement is either:

(i) a written statement that the person has signed or otherwise adopted or approved; or

Eine frühere Erklärung ist eine schriftliche Erklärung, die eine Person unterzeichnet hat oder anderweitig angenommen oder genehmigt hat.

(ii) a contemporaneous stenographic, mechanical, electrical, or other recording - or a transcription of it - that recites substantially verbatim the person's oral statement.

Bei unternehmensinternen Untersuchungen werden insbesondere die Fragen des Rechtsanwalts und die Antworten des Interviewten festgehalten und nicht nur mentale Eindrücke, Schlussfolgerungen oder den Rechtsstreit betreffende Einschätzungen des Vernehmers. Das war bei den DP-Mitschriften schon deshalb ausgeschlossen, weil der vernehmende Rechtsanwalt gar nicht selbst mitgeschrieben hat; das war Aufgabe des 2. Rechtsanwalts, der nur mitschrieb, ohne in die Befragung einzugreifen.

Damit unterliegen dessen Mitschriften nicht dem ,work-product privilege“ aus Rule 26 Federal Rules of Civil Procedure und wären „damit dem ungestörten Zugriff der Ermittlungsbehörden ausgesetzt" (Wewerka, Internal Investigations, S. 102; siehe auch Partsch, The Foreign Corrupt Practices Act der USA: das amerikanische Bestechungsverbot und seine Auswirkungen auf Deutschland, Berlin 2007, S. 61).

Die Schlussfolgerung Wewerkas, „Statt der Anfertigung eines Protokolls werden daher oftmals nur als Memo bezeichnete Notizen erstellt", wäre sicher die rechtlich korrekte Reaktion gewesen. Nach dem oben dargestellten Interviewablauf stellt sich jedoch die Frage, warum für die Erstellung eines derart unverbindlichen Memos ein Rechtsanwalt beauftragt wird, dessen einzige Aufgabe es ist, während der gesamten Befragung die „mentalen Prozesse“ seines Anwaltskollegen unentwegt in den PC zu tippen? Zumindest nach deutschem Rechtsverständnis kommt 
es für die rechtliche Qualifizierung eines Schriftstücks nicht auf dessen von einer Privatperson gewählte Bezeichnung an, sondern vielmehr auf dessen Inhalt. Ein Kaufvertrag wird nicht zu einem Mietvertrag, wenn die Überschrift in Mietvertrag geändert wird. Es ist wohl auch nicht auszuschließen, dass die amerikanischen Anwälte den Inhalt ihrer Mitschriften selbst als nicht dem work-product privilege unterliegend ansehen. Dann ist es nur konsequent, Interviewten die Einsichtnahme in die „,mentalen Mitschriften“ zu verweigern, damit sie nicht feststellen können, dass es sich - anders als behauptet - um im Rechtsverkehr übliche Protokolle handelt.

X. Da der Arbeitgeber seine Arbeitnehmer ausdrücklich verpflichtet hat, mit der von ihm beauftragten Anwaltskanzlei voll zu kooperieren, muss deren Verhalten - anders als das Verhalten der Staatsanwaltschaft - auch arbeitsrechtlichen

\section{Maßstäben entsprechen.}

Deshalb stellt sich die Frage, ob die Mitschriften der Rechtsanwälte Teil der Personalakte sind. Wenn das der Fall wäre, könnte der Arbeitnehmer über sein Einsichtsrecht in die Personalakte nach $\S 83$ Abs. 1 Satz 1 Betriebsverfassungsgesetz Kenntnis vom Inhalt der mit ihm geführten Interviews nehmen. Durch die Kenntnis seiner Personalunterlagen soll dem Arbeitnehmer das Gefühl genommen werden, Objekt undurchsichtiger (auch unternehmens)fremder Beurteilung zu sein. Außerdem soll er die Möglichkeit erhalten, sich gegen unzutreffende Angaben zu wehren (GK-BetrVG/Wiese, Vor $\S 81$ Rn. 15). Das setzt die Offenlegung aller Vorgänge voraus, die einen bestimmten Arbeitnehmer in Bezug auf sein Arbeitsverhältnis betreffen. Der Arbeitnehmer soll sich Kenntnis über alle personenbezogenen Daten verschaffen können, die der Arbeitgeber über ihn sammelt (Richardi/Thüsing, § 83 BetrVG Rn. 4). Grundlage ist letztlich das allgemeine Persönlichkeitsrecht (MünchArbR/Blomeyer, § 98 Rn. 3).

Ganz in diesem Sinne definiert das Bundesarbeitsgericht den Begriff der Personalakte in ständiger Rechtsprechung als eine „Sammlung von Urkunden und Vorgängen, die die persönlichen und dienstlichen Verhältnisse des Arbeitnehmers betreffen und in einem inneren Zusammenhang mit dem Dienstverhältnis stehen“" (BAG 25.2.1959, 4 AZR 549/57, AP Nr. 6 zu § 611 BGB Fürsorgepflicht; BAG 25.4.1972, 1 AZR 322/71, AP Nr. 9 zu § 611 BGB Öffentlicher Dienst; BAG 9.2.1977, 5 AZR 2/76, AP Nr. 83 zu § 611 BGB Fürsorgepflicht; BAG 13.4.1988, 5 AZR 537/86 AP Nr. 100 zu § 611 BGB Fürsorgepflicht).

Personalakten im Sinne des $\S 83$ BetrVG sind zunächst Unterlagen über den Arbeitnehmer, die der Arbeitgeber und die von ihm beauftragten Personalverantwortlichen und Führungskräfte selbst als Personalakte bezeichnen. Die Rechtsanwaltskanzlei hat die Personalaktentauglichkeit ihrer Mitschriften verneint, da es sich bloß um persönliche Notizen handle, die nicht an Dritte weitergegeben werden. Das steht jedoch ihrer Qualifikation als Teil der Personalakte nicht entgegen, zumal auch die Nicht-Weitergabe an Dritte (zumindest für den Arbeitnehmer) 
nicht kontrollierbar ist. Entscheidend ist der Inhalt der Mitschriften. Sobald es sich um eine Fixierung von Vorgängen handelt, die die persönlichen und dienstlichen Verhältnisse des Arbeitnehmers betreffen und die in einem inneren Zusammenhang mit dem Dienstverhältnis stehen, gehören die Protokolle zur Personalakte im Sinne des $\S 83$ BetrVG. Damit sind die Mitschriften der Rechtsanwälte Teil der Personalakte. Daran ändert auch nichts, dass sie nicht in und von der Personalabteilung verwahrt werden und nicht von Firmenangehörigen erstellt wurden, sondern deren Erstellung auf Firmenexterne outgesourct wurde.

Da auch eine freiheitliche Grundordnung keine schrankenlosen Befugnisse anerkennen kann, könnte auch das Akteneinsichtsrecht Beschränkungen unterliegen. Das Akteneinsichtsrecht darf weder rechtsmissbräuchlich (§ 241 BGB) noch schikanös (§ 226 BGB) noch zur Unzeit ausgeübt werden (GK-BetrVG/ Wiese/Franzen, § 83 Rn. 36). Dies ist bei einer unmittelbar im Anschluss an eine Befragung erbetenen Protokolleinsicht nicht ersichtlich. Ganz im Gegenteil, das Bedürfnis des Arbeitnehmers, vom Einsichtsrecht Gebrauch zu machen, besteht hier in besonderer Weise, da die Angaben der Befragten nicht von einer rechtsunkundigen Person (selbst bei Gerichten sind Protokollführer üblicherweise keine Volljuristen) protokolliert werden, die die Vorgeschichte nicht genauer kennt und daher als „,neutraler Protokollführer“ besondere Gewähr für eine unvoreingenommene Mitschrift bietet. Protokollführer ist vielmehr ein mit der Materie bestens vertrauter Rechtsanwalt, der die Antworten sogleich in einen größeren Zusammenhang stellen kann und deshalb nicht mehr neutral ist. Hinzu kommt, dass die Mitschriften in englischer Sprache abgefasst werden und die nicht auszuschließende Gefahr von Übersetzungsfehlern (siehe oben 2.2.5) besteht, die sich vermeiden ließen, wenn die Befragten Einblick in die Protokolle erhalten würden. An dieser Richtigstellung müsste dem Arbeitgeber - schon aus Gründen der Fürsorgepflicht, die ihn gegenüber seinen Arbeitnehmern trifft - besonders gelegen sein.

XI. Ein Arbeitgeber hat keinen allgemeinen Auskunftsanspruch gegenüber seinen Arbeitnehmern (Sieg, Aufklärungsinteresse versus arbeitsrechtliches Schutzgebot bei Compliance-Untersuchungen, S. 147). Arbeitnehmer können aber im Rahmen von Compliance-Untersuchungen in beschränktem Umfang zur Auskunft verpflichtet sein, nämlich dann, wenn Fragen ihren Arbeitsbereich betreffen (§ 666 BGB i.V.m. § 675 BGB). So muss z. B. ein Produktmanager darüber Auskunft erteilen, ob er mit einem Mitwettbewerber Kartellabsprachen über die Marktpreise getätigt hat (Diller, DB 2004, 313, 315). Arbeitnehmer sind auch verpflichtet, Auskunft über Wahrnehmungen im Zusammenhang mit ihrer Arbeitsleistung zu erbringen (Göpfert/Merten/Siegrist, NJW 2008, 1703, 1705; Mengel/ Ullrich, NZA 2006, 240, 243; Rudkowski, NZA 2011, 612, 613).

Über Wahrnehmungen des Arbeitnehmers außerhalb seines Arbeitsbereichs, z. B. bei Gelegenheit einer Dienstreise oder bei allgemeiner Aufklärung 
von Eigentumsdelikten (Reichold in: MünchArbR, § 49 Rn. 7), darf der Arbeitgeber nur eingeschränkt Auskunft verlangen. Hier geht es nicht um Aufgaben, die dem Arbeitnehmer übertragen wurden.

Trotzdem kann sich aus der Treuepflicht des Arbeitnehmers gegenüber seinem Arbeitgeber ( $\S 611,241$ Abs. 2 BGB), wonach er auf dessen Rechte, Rechtsgüter und Interessen Rücksicht zu nehmen hat, eine Auskunftspflicht ergeben (Böhm, Non-Compliance und Arbeitsrecht, S. 151; Dann/Schmidt, NJW 2009, 1851, 1852; Fritz/Nolden, CCZ 2010, 170, 171; Göpfert/Merten/Siegrist, NJW 2008, 1703, 1705; Greeve/Tsambikakis in: Knierim/Rübenstahl/Tsambikakis, Internal Investigations, Kap. 17 Rn. 18; Mengel, Compliance und Arbeitsrecht, Kap. 4 Rn. 21; Mengel/Ullrich, NZA 2006, 240, 243; Müller-Bonanni, AnwB1 2010, 651, 653; Rudkowski, NZA 2011, 612, 613 f.; Schürrle/Olbers, CCZ 2010, 178; Vogt, NJOZ 2009, 4206, 4213; Wastl/Litzka/Pusch, NStZ 2009, 68, 70).

Knauer (in.: Verhandlungen des 67. Deutschen Juristentages, L 161) sieht die Chance des Einzelnen bei umfassenden Befragungen wirklich frei zu entscheiden im Sinne eines: ,Nein ich möchte schweigen, ich möchte nicht kooperieren' „aufgrund der wirtschaftlichen Bedrängnis, in die er gerät, gegen null“".

Bei drohenden erheblichen Schäden (behördliche Maßnahmen, Gerichtsverfahren, Zwangsmaßnahmen) für den Arbeitgeber, ist der Arbeitnehmer nicht nur zur Beantwortung von Fragen verpflichtet, er muss sogar ungefragt von sich aus auf diese Gefahr hinweisen (Dann/Schmidt, NJW 2009, 1851, 1852; Diller, DB 2004, 313, 314; Fritz/Nolden, CCZ 2010, 170, 172; Linck in: Schaub, ArbR, § 45 Rn. 65; Joussen in: Rolfs/Giesen/Kreikebohm/Udsching, BeckOK ArbR, § 611 BGB Rn. 387; Lützeler/Müller-Sartori, CCZ 2011, 19; Mengel, Compliance und Arbeitsrecht, Kap. 1 Rn. 122; Mengel/Ullrich,NZA 2006, 240, 243; Müller-Glöge in: MüKo BGB - Band 4, § 611 Rn. 1082; Preis in: ErfK, § 611 BGB Rn. 741; Reichold in: MünchArbR, § 49 Rn. 8; Schulz, BB 2011, 629, 631; Zimmer/Stetter, BB 2006, 1445, 1451).

XII. Will die Unternehmensleitung die im Rahmen interner Ermittlungen gewonnenen Beweise für ein späteres zivilrechtliches und/oder strafrechtliches Gerichtsverfahren verwenden, empfiehlt es sich, die Aussagen der befragten Mitarbeiter umfassend zu protokollieren und die Richtigkeit des Protokolls vom Mitarbeiter nach Beendigung der Befragung durch Unterschreiben des Protokolls bestätigen zu lassen. Auf dem Weg zu diesen Aussagen sollte es vermieden werden, durch Drohung mit einer (ggf. auch rechtmäßigen) Kündigung oder mit Einschaltung der Staatsanwaltschaft (dazu, ob Verhöre bei der Staatsanwaltschaft für den Mitarbeiter immer unangenehmer sein müssen als unternehmensinterne Interviews siehe oben 2.2.4) unnötig Druck aufzubauen. Damit kann einer Rüge gegen die Reichweite und die Überzeugungskraft, das heißt den Beweiswert von privaten Ermittlungsberichten vorgebeugt werden. Zwar dürfen die Protokolle in- 
terner Ermittlungen wegen des Unmittelbarkeitsgrundsatzes $\S 250$ Satz 2 Strafprozessordnung (S. dazu Diemer in: Karlsruher Kommentar zur Strafprozessordnung..., 6. Auflage, München 2008, § 250 Rn. 1; Velten in: Systematischer Kommentar zur Strafprozessordnung, 3. Auflage Stand: 64. Lieferung Oktober 2009, Vor $\S 250$ Rn. 7 ff.) nicht als Urkundenbeweis in einen späteren Strafprozess eingeführt werden, deren Inhalt kann aber im Wege eines Vorbehalts als Vernehmungsbehelf oder allgemein zu Beweiszwecken bei der späteren Befragung des Zeugen/Angeklagten im Rahmen der Hauptverhandlung verwendet werden ( $\mathrm{We}$ werka, Internal Investigations, S. 151; BGHSt 20, 160, 161; 21, 285, 286; 34, 231, 235. Zu Begriff, Zweck und Beweiswert des Vorbehalts siehe Diemer in: Karlsruher Kommentar zur Strafprozessordnung..., 6. Auflage, München 2008, § 249 Rn. 41 ff., § 250 Rn. 2, 16 und Mosbacher in Löwe/Rosenberg, Die Strafprozessordnung und das Gerichtsverfassungsgesetz, Großkommentar, 26. Auflage, Berlin 2008, § 249 Rn. 92 ff.).

Sollen unternehmensinterne Protokolle auch gegenüber den Strafverfolgungsbehörden verwendet werden, müssen die Aussagen der Mitarbeiter so vollständig wie möglich dokumentiert werden, um dem Wahrhaftigkeitsgebot zu genügen (Dahs, Handbuch des Strafverteidigers, Rn. 310, 7. Auflage, Köln 2005; Dallmeyer in: Heghmanns/Scheffler, Handbuch zum Strafverfahren, B. II. Rn. 372, München 2008).

Die obigen Ausführungen spielten für die Interviews der Rechtsanwaltskanzlei bei den Untersuchungen im Siemens-Konzern keine Rolle, da deren Zielrichtung nicht die Strafverfolgung gegen einzelne Mitarbeiter war, vielmehr sollte das weltweite Bestechungssystem als solches aufgedeckt und "trockengelegt“ werden.

Andererseits verlangten die amerikanischen Behörden von der Unternehmensleitung arbeitsrechtliche Konsequenzen gegen nicht kooperierende Mitarbeiter (Talk or Walk) aber auch die Sanktionierung von Mitarbeitern, die Teil des Korruptionssystems waren. Die Anwaltskanzlei musste diesen regelmäßig Listen über als Konsequenz der Untersuchungen gekündigte Mitarbeiter vorlegen.In Deutschland gibt es aber keine „Hire and Fire-Mentalität“, sondern einen umfassenden gesetzlichen Kündigungsschutz (Kündigungsrecht ist kein Strafrecht, sondern Zivilrecht. Die weitaus überwiegende Beendigung von Arbeitsverhältnissen erfolgt in Deutschland durch Aufhebungsverträge, oft mit Abfindungszahlungen ,vergoldet"). Um diesem zu genügen muss der Arbeitgeber die behaupteten Verstöße beweisen können. Und dafür wäre das oben aufgezeigte Verfahren sehr hilfreich. Verdachtskündigungen wegen vermuteter Compliance-Verstöße sind zwar grundsätzlich möglich, unterliegen aber strengen richterrechtlichen Voraussetzungen.

XIII. Das Verhältnis von Privatermittlern und Staatsanwaltschaft wurde vom $D P$-Chef wie folgt beschrieben: „Die Legalität der Untersuchung steht au- 
Ber jedem Zweifel. Die gesamte Untersuchung von $D P$ ist in voller Kenntnis und Übereinstimmung mit der Münchener Staatsanwaltschaft durchgeführt worden.“

Mancher Interviewte sah in dieser Äußerung eine Bestätigung, dass auch der Inhalt seines Interviews (dessen Einsicht ihm verweigert wurde) bei der Staatsanwaltschaft zu deren „,voller Kenntnis“ gelandet ist. Dass umgekehrt die Inhalte von Protokollen der Staatsanwaltschaft bei der Rechtsanwaltskanzlei gelandet sind, wurde dem Interviewten spätestens dann klar, wenn er gefragt wurde, wie denn seine bei der Staatsanwaltschaft getätigte Äußerung ,...“ zu verstehen sei?

Von vielen Befragten wurden die Privatermittler als Hilfsorgan der deutschen Staatsanwaltschaft wahrgenommen. Tatsächlich sollen nach ,anfänglichen Diskrepanzen zwischen den internen Ermittlern von $D P$ und den Münchner Staatsanwälten beide Seiten im weiteren Verlauf des Verfahrens derart eng zusammengearbeitet haben, dass ein ständiger gegenseitiger Austausch über den Fortschritt der Ermittlungen stattgefunden hat und Ermittlungsschritte koordiniert wurden“ (Wewerka, Internal Investigations, S. 122; OLG München, NZG 2009, 665, 666; Hamm, NJW 2010, 1332, 1335). Diese Zusammenarbeit soll im Fall Ferrostaal 2010 so weit gegangen sein, dass die Staatsanwaltschaft ,über einen Mittelsmann“ der Unternehmensleitung mitgeteilt hat, dass es „vielleicht sinnvoll sei“" statt ihrer bisherigen wenig kooperationsbereiten Kanzlei doch „DP mit den internen Ermittlungen zu beauftragen. Siemens und auch die Staatsanwaltschaft hätten gute Erfahrungen mit den Amerikanern gemacht." (manager magazin vom 20.11.2011, Korruptionsjäger Debevoise\&Plimpton, Angst \& Schrecken) — was dann auch umgehend geschah. Die enge Zusammenarbeit mit $D P$ hatte für die Staatsanwaltschaft den großen Vorteil, dass sie an Beweismaterial gekommen ist, „,das sie ohne die $D P$-Ermittler gar nicht hätte erlangen können. Denn anders als den Münchener Staatsanwälten waren den Anwälten von $D P$ keine territorialen und finanziellen Grenzen gesetzt: Sie ermittelten auch in Russland, China und im Nahen Osten“ (Wewerka, Internal Investigations, S. 123; Hildebrand, Welt am Sonntag vom 21.9.2008). „Beispielsweise entsendete DP rund 40 Ermittler nach China, die dort sämtliche Unternehmensunterlagen von Siemens East Asia screenten und zahlreiche Mitarbeiter interviewten. Diese Möglichkeiten bleiben den deutschen Staatsanwälten aufgrund des Territorialitätsprinzips verwehrt und hätten allenfalls im Wege der Amtshilfe wahrgenommen werden können“ (Wewerka, Internal Investigations, S. 123 Fn. 53; vgl. ferner Pfrode in: Strafverteidigung im Rechtsstaat, S. 740, 745, sowie Salvenmoser/Schreier in: Achenbach/ Ransiek, Hdb WiStra, Kap. XV Rn. 34).

Der Fall Siemens blieb kein Einzelfall. Nach Abschluss der dortigen Untersuchungen gab es umfassende interne Sachverhaltsaufklärung durch Privatpersonen, überwiegend durch darauf spezialisierte amerikanische Kanzleien, auch in anderen Unternehmen unter anderem bei der MAN SE, der Porsche $A G$, der Daimler $A G$, der Ferrostaal $A G$ und der Volkswagen $A G$. Auch in diesen Fällen 
gab es eine enge Zusammenarbeit zwischen Privatermittlern und Staatsanwaltschaft.

Die deutschen Strafverfolgungsbehörden dürfen gemäß $\S 152$ Abs. 2, 160 Abs. 1 Strafprozessordnung grundsätzlich nur bei Vorliegen eines Anfangsverdachts im Hinblick auf eine konkrete Straftat tätig werden (Löwe/Rosenberg, Die Strafprozessordnung und das Gerichtsverfassungsgesetz, Großkommentar: Beulke, § 152 Rn. 22 und Erb, § 160 Rn. 19). Ein solcher Anfangsverdacht setzt zureichende tatsächliche Anhaltspunkte (konkrete Tatsachen, die auf die Begehung einer Straftat hindeuten) voraus, die nach kriminalistischer Erfahrung die Beteiligung des Betroffenen an einer verfolgbaren strafbaren Handlung als möglich erscheinen lassen. Bloße Vermutungen oder kriminalistische Hypothesen reichen dagegen nicht (zum Begriff des Anfangsverdachts siehe Beulke, Strafprozessrecht, 11. Auflage 2010, § 152 Rn. 34).

Die $S E C$ empfiehlt den Unternehmen (und erwartet es wohl auch von diesen) die Durchführung einer internen Investigation schon beim bloßen Vorliegen eines sehr allgemeinen „Verdachts auf Unregelmäßigkeiten“.

Obwohl die Verdachtsmomente, denen die Staatsanwaltschaft München ursprünglich nachging, sich nur auf einige wenige Unternehmensbereiche der Siemens AG bezogen, erteilte die Unternehmensleitung $D P$ den Auftrag, eine von den Erkenntnissen der Staatsanwaltschaft unabhängige vollständige Untersuchung vorzunehmen: „Vollständig“ heißt breit angelegt und konsequent. Die Untersuchung ist nicht beschränkt auf Vorwürfe im Zusammenhang mit dem Ermittlungsverfahren der Münchener Staatsanwaltschaft. Sie kann jeden Bereich, jede Region, jede Zentrale Einheit, jedes Projekt und jede Person einschließen. Maßgeblich ist allein die Aufklärung der Fakten." (so der Vorsitzende des Prüfungsausschusses der Siemens $A G$ auf der Siemens Hauptversammlung am 25.1.2007). Auf die Frage von Führungskräften, was das für die Umsetzung im Unternehmensalltag bedeutet, antwortete der DP-Deutschlandchef Thomas Schürrle: „Wir werden jeden Stein auch in Timbuktu umdrehen. Sie werden das Unternehmen danach nicht mehr wiedererkennen“".

„Der Umstand, dass die Münchner Staatsanwaltschaft das im Rahmen der Internal Investigations zusammengetragene Beweismaterial in der Folge gezielt für die Einleitung von entsprechenden Strafverfahren gegen verdächtige Mitarbeiter verwendet hat, stellt daher im Ergebnis eine Umgehung des Erfordernisses des Anfangsverdachts dar. Ohne die Unterstützung von Debevoise wäre die Münchner Staatsanwaltschaft nicht auf belastendes Material gestoßen, da sie entsprechende Ermittlungen mangels Anfangsverdachts gar nicht erst hätte aufnehmen dürfen" (Wewerka, Internal Investigations, S. 205 f.; vgl. Böse, ZStW 119 [2007], 848, 862).

Der Weitergabe von Erkenntnissen aus unternehmensinternen Untersuchungen an Staatsanwaltschaft und andere staatlichen Behörden sind keine gesetzlichen Grenzen gesetzt (Diller, DB 2004, 313, 319; Theile, StV 2011, 381, 382). In 
der Unternehmenspraxis werden die Untersuchungsergebnisse auch regelmäßig an in- und ausländische Behörden weitergeleitet, um mit einer uneingeschränkten Kooperationsbereitschaft Vergünstigungen bei eventuellen Sanktionen (Bußgeldund Schadensersatzhöhe, Vermeidung eines Ausschlusses von öffentlichen Aufträgen etc.) zu erhalten. Es gibt keinen arbeitsrechtlichen Grundsatz, der es einem Arbeitgeber verbietet, von einem Arbeitnehmer selbstbelastende Aussagen zu verlangen (Diller, DB 2004, 313, 319). Nachdem sich diese Rechtslage zwischenzeitlich in den Unternehmen herumgesprochen hat, können sich Arbeitnehmer immer häufiger nicht mehr an sie belastende Umstände erinnern (Dann/Schmidt, NJW 2009, 1851, 1853; Müller-Bonanni, AnwB1 2010, 651, 653; Scharnberg, Illegale Internal Investigations, S. 272; Wisskirchen/Glaser, DB 2011, 1392, 1447; Zimmer, ZRFC 2011, 259, 260).

Im Strafprozess ist der Arbeitnehmer dagegen berechtigt, die Aussage zu verweigern. Er kann sich als Zeuge bei Selbstbelastungsgefahr auf sein Auskunftsverweigerungsrecht nach $\S 55$ Abs. 1 StPO beziehungsweise als Beschuldigter auf sein Aussageverweigerungsrecht nach $\S 136$ Abs. 1 Satz 2 StPO berufen.

Unternehmensinterne Untersuchungen bringen zahlreiche Probleme mit sich, weil deren Untersuchungsergebnisse an in- und ausländische Strafverfolgungsbehörden weitergegeben werden, ohne dass auf der Stufe der Beweismittelgewinnung die Vorschriften zum Rechtshilfeverkehr oder die Formvorschriften der StPO für staatliche Ermittlungen eingehalten werden müssen. Das birgt die Gefahr, dass umfassende interne Untersuchungen ,sich als rechtswidrige Parallelverfahren zur StPO etablieren könnten“. Deshalb fordert Wewerka (Internal Investigations, S. 211), dass der drohenden Umgehung elementarer Verfahrensvorschriften angemessen begegnet wird, siehe hierzu meine Vorschläge unten.

XIV. War die Auswirkung der Zusammenarbeit von Privatermittlern und deutscher Staatsanwaltschaft auf das berufliche Schicksal für einige Doppelbefragte schon schwierig einzuschätzen, so zeigte folgende Aussage des Deutschland-Chefs von DP Thomas Schürrle, dass die Befragungen durch die Privatermittler-anders als durch staatliche deutsche Organe - für sie auch einen internationalen Charakter haben: „,DP berichtet $1 \mathrm{x} /$ Woche an den Vorsitzenden des Prüfungsausschusses des Aufsichtsrats und telefonisch an die US-Behörden, und $1 \mathrm{x} /$ Monat erfolgt ein persönliches Treffen beim DoJ und der SEC. Die Berichte - sowohl an den Prüfungsausschuss als auch an die $S E C$ - erfolgen fast ausschließlich mündlich, am Ende der Ermittlungen gibt es eher keinen großen schriftlichen Bericht sondern meist zusammenfassende Folien.“

Privatermittler als Hilfsorgan ausländischer Behörden (Departement of $\boldsymbol{J} u$ stice $=$ Justizministerium, United States Securities and Exchange Commission = Börsenaufsichtsbehörde für die Kontrolle des Wertpapierhandels in den Vereinigten Staaten) gegen Inländer? 
Einige Befragte waren verunsichert, wie sich die Zusammenarbeit „deutsche Staatsanwaltschaft - Privatermittler - amerikanische Behörden“" auf ihr Arbeitsverhältnis in Deutschland auswirkt und ob sie bei mangelnder Kooperationsbereitschaft gegebenenfalls mit Problemen bei der nächsten Einreise in die USA rechnen müssen.

Manche Mitarbeiter hatten auch ein mentales Problem damit, dass die Privatermittler die aus ihren Büros (in Kopie) mitgenommenen Unterlagen zur elektronischen Auswertung ins Ausland verbracht haben. Begründung: In Deutschland gäbe es keine hierzu fähigen Dienstleister...

Gegenüber der deutschen Staatsanwaltschaft hätte man ein derartiges Verhalten nicht akzeptiert, da das Territorialitätsprinzip auch für den Auswertungsort von im Inland beschlagnahmten Unterlagen gilt.

XV. Um die vollständige und zeitnahe Aufklärung von möglichen Compliance-Verstößen zu erleichtern und zu beschleunigen haben sich in der Unternehmenspraxis Amnestieangebote bewährt.

Beispiel: „Das Unternehmen wird gegenüber Arbeitnehmern, die bis zum [...] das Unternehmen freiwillig und wahrheitsgemäß und nach dem Wissen des Arbeitnehmers vollständig über mögliche Verstöße gegen die Gesetze zum Verbot der Korruption im Öffentlichen Sektor, einschließlich jeglicher Unregelmäßigkeiten bei der Buchführung, informieren, keine Schadensersatzansprüche geltend machen und das Beschäftigungsverhältnis nicht einseitig lösen. Dieses Amnestieangebot gilt grundsätzlich für alle Mitarbeiter, mit Ausnahme der Vorstandsmitglieder/Geschäftsführer, Zentralabteilungsleiter und Bereichs-/Geschäftsgebietsleiter sowie [...]. Das Unternehmen behält sich leichtere Disziplinarmaßnahmen wie Abmahnung, Versetzung, Änderungen im Verantwortungsbereich oder zusätzliches Compliance-Training vor.“

Der Arbeitgeber hat es naturgemäß in der Hand, dass er auf arbeitsrechtliche Maßnahmen gegen aussagebereite Mitarbeiter (weitgehend) verzichtet oder bei Privatklagedelikten auf die Stellung eines Strafantrags.

Soweit seine Mitarbeiter jedoch Offizialdelikte begangen haben, darf er nicht den Eindruck erwecken, dass sein Amnestieangebot auch staatliche Strafverfolgungsbehörden bindet.

Die Fürsorgepflicht verlangt vom Arbeitgeber eine diesbezügliche Aufklärung, die einerseits der Wahrheit entsprechen muss, anderseits aber auch nicht zu Aussage-abschreckend formuliert sein sollte.

Beispiel: „Das Amnestieangebot des Unternehmens bindet in keiner Weise staatliche Strafverfolgungs- oder sonstige Ermittlungsbehörden. Das Unternehmen kann Arbeitnehmer nicht davon freistellen, dass Ermittlungen von staatlichen Behörden eingeleitet/durchgeführt werden. Jedoch wird das Unternehmen im Fall von strafrechtlichen Ermittlungen gegen Arbeitnehmer, die Gebrauch von 
der Amnestieregelung gemacht haben, die ermittelnden staatlichen Behörden auf die Kooperation des Arbeitnehmers mit dem Unternehmen hinweisen und darauf hinwirken, dass die Kooperation entsprechend gewürdigt wird. Die staatlichen Behörden sind aber in ihrer Entscheidung selbstverständlich frei, ob sie die Kooperation des Arbeitnehmers berücksichtigen. Nach den bisherigen Erfahrungen ist das Unternehmen jedoch zuversichtlich, dass zahlreiche Strafverfolgungs- und sonstige Ermittlungsbehörden eine solche Kooperation vorteilhaft aufnehmen.“

Gerichte haben bei der Strafzumessung nach $\S 46$ Abs. 2 Strafgesetzbuch grundsätzlich auch das Verhalten des Täters nach der Tat strafmildernd zu berücksichtigen, also auch eine umfassende Aussage bei unternehmensinternen Untersuchungen.

Bei einem kooperierenden Täter können die Strafgerichte die Strafe nach $\S 46 \mathrm{~b}$ Strafgesetzbuch (Kronzeugenregelung) mildern oder auch ganz von Strafe absehen, wenn dieser „durch freiwilliges Offenbaren seines Wissens wesentlich dazu beigetragen hat, dass eine Tat nach $\S 100 \mathrm{a}$ Abs. 2 der Strafprozessordnung aufgedeckt werden konnte [...]“ oder „freiwillig sein Wissen so rechtzeitig einer Dienststelle offenbart, dass eine Tat nach $\S 100 \mathrm{a}$ Abs. 2 der Strafprozessordnung, von deren Planung er weiß, noch verhindert werden kann [...]". Als Vorschriften des $\S 100 \mathrm{a}$ Abs. 2 Strafprozessordnung kommen im Zusammenhang mit internen Untersuchungen insbesondere folgende in Betracht: § 108e Strafgesetzbuch (Bestechlichkeit und Bestechung von Mandatsträgern), § 298 Strafgesetzbuch (Wettbewerbsbeschränkende Ausschreibungen), § 299 Strafgesetzbuch (Bestechlichkeit und Bestechung im geschäftlichen Verkehr) und § 334 Strafgesetzbuch (Bestechung von Amtsträgern und Europäischen Amtsträgern).

XVI. Die Unternehmensleitung der Siemens $A G$ erwartet von ihren Mitarbeitern, dass sie Hinweise auf drohende oder bestehende Compliance-Fälle unverzüglich melden. Arbeitnehmer, die Compliance-Verstöße feststellen, können diese firmenoffen ihrer Führungskraft oder der Compliance-Organisation, aber auch verdeckt/anonym über ein Compliance-Helpdesk im „Tell us“-Bereich (Webseite und ein Call-Center durch einen auf Compliance-Themen spezialisierten externen Anbieter) oder einem externen Ombudsmann mitteilen.

Jegliche Art von Vergeltungsmaßnahmen gegen Personen, die in gutem Glauben Compliance-Fälle gemeldet haben, wird von der Unternehmensleitung nicht toleriert. Das Verbot von Vergeltungsmaßnahmen bezieht sich dabei auf jede Maßnahme, die direkt oder indirekt einen negativen Einfluss auf das Arbeitsverhältnis, Verdienstmöglichkeiten, Bonuszahlungen, Karrieremöglichkeiten oder andere arbeitsbezogene Interessen des Meldenden haben kann.

Die Compliance-Organisation will ausdrücklich alle Meldenden schützen, wenn sie in gutem Glauben Compliance-Fälle von Kollegen gemeldet haben. Damit soll Arbeitnehmern die Angst genommen werden, unrechtmäßiges Kol- 
legenverhalten zu melden, auch wenn es nur eine Vermutung ist. Das deutsche Strafrecht hat allerdings den Schwerpunkt des Persönlichkeitsschutzes bei nicht nachweisbaren ehrenrührigen Behauptungen anders gesetzt:

\$ 186 (üble Nachrede) StGB: Wer in Beziehung auf einen anderen eine Tatsache behauptet oder verbreitet, welche denselben verächtlich zu machen oder in der öffentlichen Meinung herabzuwürdigen geeignet ist, wird, wenn nicht diese Tatsache erweislich wahr ist, mit Freiheitsstrafe bis zu einem Jahr oder mit Geldstrafe $[\ldots]$ bestraft.

Das heißt, wenn ein Arbeitnehmer einen Compliance-Verstoß eines Kollegen meldet, der nicht bewiesen werden kann, erfüllt er den Tatbestand der üblen Nachrede, selbst wenn die behauptete Tatsache (Compliance-Verstöße sind grundsätzlich geeignet, einen Menschen in der öffentlichen Meinung herabzuwürdigen) tatsächlich stimmt. Das deutsche Strafrecht will also verhindern, dass ein Beschuldigter beweisen muss, dass er etwas nicht getan hat (Grundsatz: Im Zweifel zu Gunsten des Angeklagten/Beschuldigten).

Will der Arbeitgeber sich nicht dem Verdacht aussetzen, seine Mitarbeiter zur üblen Nachrede anzustiften, muss er auf die Gefahr einer Strafbarkeit nicht nachweisbarer Anschuldigungen hinweisen. Der in den Compliance-Richtlinien enthaltene Hinweis „Es ist verboten, wider besseren Wissens Mitarbeiter (Kollegen, Führungskräfte) zu verdächtigen" ist aber nicht ausreichend; er warnt nur vor einem Verstoß gegen $§ 187$ StGB (Verleumdung: Wer wider besseres Wissen in Beziehung auf einen anderen eine unwahre Tatsache behauptet oder verbreitet, welche denselben verächtlich zu machen oder in der öffentlichen Meinung herabzuwürdigen oder [...] geeignet ist, wird mit Freiheitsstrafe bis zu zwei Jahren oder mit Geldstrafe [...] bestraft).

Whistleblowen hat sich als erfolgversprechendes Instrument zur Aufdeckung und Bekämpfung von Compliance-Verstößen erwiesen. Vor allem, wenn es anonym erfolgt, und es um kleinere, eher formale Verstöße geht, kann es allerdings die unangenehme Nebenwirkung einer Vergiftung des Betriebsklimas haben (es könnte ja jeder der anonyme Hinweisgeber gewesen sein). Eine bislang lockere Vertrauenskultur kann schnell in eine Misstrauenskultur umkippen. Petzen von tatsächlich begangenen Regelverstößen von Mitschülern war schon in der Schule nicht stimmungsfördernd, gleiches galt auch unter Geschwistern in der Familie. Anonyme Anzeigen haben in Ländern mit Diktaturerfahrung (wie z. B. Polen und Deutschland, und hier insbesondere Ostdeutschland) eine ganz andere soziale Wahrnehmung als z. B. in den Vereinigten Staaten.

Ein beschuldigter Arbeitnehmer muss über eine Anzeige, auch über eine anonyme, informiert werden. Das ist im Regelfall für eine Sachverhaltsaufklärung von Nutzen, denn nur so kann ein Beschuldigter zur schnellen Aufklärung von unberechtigten Vorwürfen beitragen und gegebenenfalls Hinweise für der Meldung zugrundeliegende mögliche niedere Beweggründe des Anzeigenden geben. 
Was darf der eines Compliance-Verstoßes Beschuldigte vom Arbeitgeber verlangen, wenn der Meldende kein Abpfeifer von Unregelmäßigkeiten, sondern ein Verpfeifer war? Wenn dieser also die Whistle-Blower-Möglichkeiten genutzt hat, um sich an dem Beschuldigten zum Beispiel für eine verpasste Beförderung, verweigerte Lohnerhöhung, ungerechte Behandlung oder verlorene Arbeitnehmerwahl zu rächen oder ihm aus persönlichen Gründen (Neid, Eifersucht) schaden will? Zu denken wäre an eine Abmahnung, in schweren Fällen auch an eine verhaltensbedingte Kündigung, zumindest aber an eine Aufforderung, sich bei dem beschuldigten Kollegen unternehmensöffentlich zu entschuldigen. Aus Gründen der Fürsorgepflicht darf der Arbeitgeber nach Feststellung unredlichen Verhaltens gegenüber einem Kollegen nicht ohne Weiteres - so als sei nichts geschehen - zur Tagesordnung übergehen. Dies fällt nach Abschluss von unternehmensinternen Untersuchungen, die keine nachweisbaren Hinweise auf unrechtmäßiges Verhalten ergeben haben, den Compliance-Organisatoren nicht immer leicht, denn „dann traut sich ja keiner mehr frei und umfassend auszupacken“".

XVII. Wenn die Staatsanwaltschaft ein Strafverfahren einstellt, wird dies dem Beschuldigten mitgeteilt. Eine entsprechende Verpflichtung gibt es bei unternehmensinternen Untersuchungen nicht. Das heißt, ein Arbeitnehmer gegen den durch das Unternehmen oder/und durch eine von diesem beauftragte externe Rechtsanwaltskanzlei ermittelt wurde, hat keinen Anspruch auf eine Entwarnung, dass er jetzt wieder ein ganz normales Mitglied der Betriebsgemeinschaft ist. Auch das betriebliche Umfeld, das die Ermittlungen mitbekommen hat, erfährt nicht, dass der Kollege Compliance-mäßig nicht weiter im Verdacht von Unregelmäßigkeiten steht. Dies hat nicht nur Auswirkungen auf das „Seelenheil“ des Betroffenen, sondern kann durchaus erhebliche materielle Nachteile mit sich bringen. Ein Kollege, der in die Mühlen der internen Investigatoren geraten ist, wird sicher nicht bei der nächsten Beförderung oder Förderungsversetzung berücksichtigt, auch die jährliche Gehalts-/Boni-Erhöhung wird (bescheidener) ausfallen. Die Führungskräfte wollen schließlich nicht in den Verdacht kommen, Compliance-Verstöße ihrer Mitarbeiter nicht ernst zu nehmen.

Ein Chief Compliance Officer hatte den Wunsch eines von Compliance-Untersuchungen Betroffenen abgelehnt, ihm eine schriftliche Erklärung zu geben, dass die langjährigen intensiven Compliance-Untersuchungen gegen ihn keinerlei Hinweise auf unrechtmäßiges Verhalten ergeben haben. Begründung: „Bisher konnte Ihnen zwar nichts nachgewiesen werden, aber es ist ja nicht ausgeschlossen, dass in der Zukunft doch noch Unregelmäßigkeiten zu Tage treten können“. Das war dem Vorstandsvorsitzenden dann doch zuviel der (vorverurteilenden) Vorsicht. Er vereinbarte deshalb mit dem Konzernsprecherausschuss, dass einem Mitarbeiter auf dessen Wunsch nach zweijährigen ergebnislosen Compliance-Untersuchungen folgender Exkulpationsbrief ausgestellt wird: 
„Sehr geehrter Herr/Frau [...],

Sie wurden im Zusammenhang mit dem Vorgang [...] von der Compliance-Organisation / Internen Revision / Rechtsanwaltskanzlei [...] zu Compliance-widrigem Verhalten befragt. Wir bedanken uns für Ihre Kooperation. Die Untersuchungen haben keine Anhaltspunkte für ein Fehlverhalten Ihrerseits ergeben. Wir bedauern die Ihnen in diesem Zusammenhang entstandenen Unannehmlichkeiten und wünschen Ihnen für Ihren beruflichen Werdegang weiterhin viel Erfolg. Unterschrift Chief Compliance Officer; Unterschrift Untersuchungsleiter“.

XVIII. Da es unternehmensinterne Ermittlungen durch externe amerikanische Anwaltskanzleien in Deutschland erst seit wenigen Jahren gibt (siehe Einführung), gibt es hierfür auch noch keine im Rechtsverkehr gewohnheitsrechtlich anerkannte (zu denken wäre - in Anlehnung an den Deutschen Corporate Governance Kodex für gute Unternehmensführung - an einen von Arbeitgeber- und Arbeitnehmervertretern sowie Rechtsanwälten zu entwickelnden Kodex für faire unternehmensinterne Untersuchungen), geschweige denn gesetzlich verankerte Verfahrensregelungen. Bislang variieren die Rechte der Befragten bei Internal Investigations stark je nach Unternehmen und Anwaltskanzlei. „Diese Delegation staatlicher Ermittlungsautorität und -verantwortung im strafrechtlichen Bereich kann man für sinnvoll halten, dies entbindet jedoch nicht davon, hierfür unter Berücksichtigung der deutschen bzw. kontinentaleuropäischen Rechtstradition die entsprechenden gesetzlichen Grundlagen und Rahmenbedingungen zu schaffen" (Wastl, Zwischenruf: Privatisierung staatsanwaltschaftlicher Ermittlungen, ZRP 2011, 57). Naheliegend wäre es, die in der deutschen Strafprozessordnung festgelegten Grundsätze staatsanwaltschaftlicher Vernehmungen (die für Private nicht unmittelbar gelten) als Mindeststandard auch für unternehmensinterne Vernehmungen festzulegen.

Also z. B. in Anlehnung an $§ \mathbf{1 3 6}$ Strafprozessordnung:

- Bei Beginn der ersten Vernehmung ist der Befragte darüber zu informieren, um welches Verhalten es geht und welche Rechtsvorschriften damit verletzt worden sein könnten.

- Der Befragte ist darauf hinzuweisen, dass es ihm im Fall der Selbstbelastung freisteht, sich zu dem Sachverhalt zu äußern oder nicht zur Sache auszusagen, ohne dass ihm das schon als Schuldeingeständnis ausgelegt wird.

- Der Befragte ist darauf hinzuweisen, dass es ihm freisteht, jederzeit, auch schon vor seiner Befragung, einen Rechtsanwalt seines Vertrauens zu befragen und zu jeder Befragung hinzuzuziehen.

In Anlehnung an § 136a Strafprozessordnung:

— Die Freiheit der Willensentschließung und der Willensbetätigung des Befragten darf nicht beeinträchtigt werden durch körperlichen Eingriff, Verabreichung von Mitteln, Quälerei, Täuschung oder Hypnose. 
— Die Drohung mit einer rechtlich unzulässigen Maßnahme (z. B. sozial ungerechtfertigte Kündigung) und das Versprechen eines gesetzlich nicht vorgesehenen Vorteils (z. B. keine Strafverfolgung durch die Staatsanwaltschaft bei Aussagebereitschaft in unternehmensinterner Befragung) sind verboten.

- Maßnahmen, die das Erinnerungsvermögen oder die Einsichtsfähigkeit des Beschuldigten beeinträchtigen sind nicht gestattet.

- Diese Verbote gelten ohne Rücksicht auf die Einwilligung des Befragten.

- Aussagen, die unter Verletzung dieser Verbote zustande gekommen sind, dürfen auch dann weder unternehmensintern noch extern verwendet werden, wenn der Befragte der Verwertung zustimmt.

In Anlehnung an $§ 147,168 \mathrm{ff}$. Strafprozessordnung $\S ~ 145,168 \mathrm{ff}$.

— Über die Befragung ist von der Befragerseite ein Protokoll zu erstellen.

- Dieses Protokoll ist den an der Befragung Beteiligten zur Durchsicht vorzulegen.

— Der Befragte erhält ausreichend Zeit dieses einzusehen.

- Bei Unrichtigkeiten hat er einen Anspruch auf sofortige Berichtigung.

- Falls der Befragte mit dem Inhalt des Protokolls einverstanden ist, sollte er dies durch seine Unterschrift bestätigen. Falls der Befragte die Unterschrift verweigert, ist der Verweigerungsgrund hierfür zu protokollieren.

XIX. Interne Ermittlungen haben sich in Deutschland nicht - wie vielfach vorausgesagt - zu einem ständig ansteigenden Massenphänomen entwickelt. Grund: Bis 1998 waren „nützliche Aufwendungen“ (so der Titel einer Siemens-Schrift zur Aufarbeitung ihrer Compliance-Vergangenheit) im Ausland zur Erlangung von Aufträgen für inländische Werke nicht nur nicht strafbar, sondern sogar steuerbegünstigt (sie konnten als Betriebsausgaben verbucht werden). Zwischenzeitlich hat sich aber aufgrund von sensationsmäßig aufgemachten Medienberichten über Korruptionsvorfälle - zumindest in deutschen Unternehmen - bei den Unternehmenslenkern und deren Vertriebsbeauftragten herumgesprochen, dass Bestechung auch im Ausland, auch wenn sie ohne direkte persönliche Bereicherungsabsicht im (falschverstandenen) Unternehmensinteresse zur Sicherung von Arbeitsplätzen im Inland geschieht und auch wenn es der internationale Wettbewerb ebenso (und geschickter) tut, strafbar ist. Mitarbeiter, die in der Vergangenheit in einem korruptionsbelasteten Umfeld (landesüblich) bestochen haben, mussten schmerzhaft erfahren, dass sie mit keiner unternehmensseitigen Unterstützung mehr rechnen konnten. Viele mussten ihr (damals gegebenenfalls sogar augenzwinkernd als „firmennützlich“ geduldetes) Verhalten, wenn nicht mit Bestrafung oder Schadensersatz, dann doch zumindest mit der Beschädigung ihrer eigenen beruflichen Integrität bezahlen. Deren Schicksal wurde von den „säubernden“ Unternehmens-Institutionen bei einschlägigen Compliance-Schulungen gerne als abschreckendes Beispiel eingehend kommuniziert. Die öffentliche Ächtung von 
Compliance-Vergehen traf allerdings nicht nur tatsächliche Täter. Mitarbeiter aus Compliance-belasteten Unternehmen, die sich extern bewerben wollten, wurden bei potenziellen neuen Arbeitgebern sofort aus dem Bewerbungsverfahren ausgesondert. Der Konzernsprecherausschuss der Siemens $A G$ sah sich deshalb veranlasst, mit der Firmenleitung zu vereinbaren, dass Mitarbeiter mit Wechselabsicht auf deren Wunsch folgende Bestätigung erhalten: „Das Ausscheiden von Herrn/ Frau [...] erfolgt auf seinen/ihren Wunsch und steht in keinerlei Zusammenhang mit den in unserem Unternehmen derzeit durchgeführten Compliance-Untersuchungen“.

Mit der Aufdeckung vieler nach der Gesetzesänderung flächendeckend angelegter „Von ganz oben angeordneter“ Bestechungssysteme ist demzufolge künftig kaum mehr zu rechnen. Nicht ausgeschlossen werden können allerdings weiterhin wirtschaftskriminelle Handlungen Einzelner. Dass Handlungsweisen nicht automatisch enden, nur weil der Gesetzgeber sie unter Strafe gestellt hat, ist aber kein Sonderproblem der Wirtschaftskriminalität, sondern ein weltweites Rechtsphänomen.

Wer vermeiden möchte, dass künftig Strafrichter bei Wirtschaftsstraftaten nur noch auf Grundlage von Akten urteilen können, die ihnen von privaten Ermittlern vorgelegt werden, muss schnell in den Ausbau von Schwerpunktstaatsanwaltschaften investieren. Die Justiz muss wieder die Ermittlungshoheit bei der Wirtschaftskriminalität zurückgewinnen. Will man das seit Jahrhunderten tradierte staatliche Straf- und Strafermittlungsmonopol wieder herstellen, dann müssen Juristen eingestellt werden, die über gute Kenntnisse des Wirtschaftslebens verfügen und deshalb mit den Wirtschaftsbossen auf „gleicher Augenhöhe“ reden können. Falls diese auf dem freien Arbeitsmarkt zu den Konditionen des öffentlichen Dienstes nicht zu erhalten sein sollten, wäre auch an den verfahrensbezogenen, zeitlich befristeten Einsatz von Beratern mit „,wirtschaftsüblicher“ Bezahlung zu denken. Diese würden dann nicht mehr nach selbst festgelegten und aus fremden Rechtskreisen importierten (mit dem deutschen Rechtsverständnis nur schwer kompatiblen) Spielregeln eigenmächtig ermitteln und in den Führungsetagen der Wirtschaft „Angst und Schrecken“ verbreiten können. Sie unterlägen als „,entliehene Aufklärer“ der Verfahrenshoheit der staatlichen Ermittlungsbehörden. Damit wäre die Einhaltung rechtsstaatlicher Vernehmungsstandards auch am Arbeitsplatz gesichert. „Warum sollte man Topmanagern Rechte verwehren, die mutmaßlichen Taschendieben oder Totschlägern zugestanden werden" (Rohwetter, DIE ZEIT Nr. 41/2015 vom 8.10.2015)?

Die Bekämpfung der internationalen Wirtschaftskriminalität darf künftig auch nicht mehr allein einem einzigen Land (auch wenn dieses aufgrund seiner politischen und wirtschaftlichen Macht dabei überaus erfolgreich war, wie die Beispiele Siemens, Volkswagen, FIFA, französische Großbank BNP Paribas zeigen) mit deren landeseigenen Spielregeln überlassen werden. Der Kampf gegen 
wettbewerbsverzerrende Korruption sollte vielmehr vordringliche Aufgabe der gesamten Staatengemeinschaft sein und durch multinationales Recht geregelt werden. Als adäquates Mittel hierfür kämen meines Erachtens Ergänzungen der am 17.12.1997 unterzeichneten Konvention zur Bekämpfung der Korruption des Rates der Organisation für Wirtschaftliche Zusammenarbeit und Entwicklung - OECD über Verfahrensregeln bei der länderübergreifenden Strafverfolgung von Wirtschaftsstraftaten in Betracht (Stichworte: Territorialitätsprinzip als Aufklärungsbremse, vereinfachte und erweiterte Amtshilfe).

Der Einsatz von Privatermittlern zusätzlich und parallel zu staatlichen Ermittlern hat sich nicht als Königsweg erwiesen, aber auch nicht als Irrweg, sondern war ein Ausweg aus der Misere, dass der Staatsanwaltschaft schlicht die Ausstattung und das Personal gefehlt hat, um die komplexen internationalen Zusammenhänge von Wirtschaftskriminalität aufzuklären. Oder um es mit den Worten des Parlamentarischen Staatssekretärs und Mitglied des Deutschen Bundestags Max Stadler auszudrücken: „Die Staatsanwaltschaft hätte in Anbetracht ihrer Ressourcen nicht ohne weiteres die Möglichkeit gehabt, die ungeheure Menge zu sichtender Unterlagen in angemessener Zeit zu bewältigen. Wir können also feststellen, dass bei Großverfahren die Arbeit einer intern ermittelnden Kanzlei, die den Strafverfolgungsbehörden relevante Dokumente übermittelt, erheblich zur Verfahrensbeschleunigung beitragen kann." Diese richtige Erkenntnis schließt jedoch nicht aus, dass die von der Politik zugegebene strukturelle Unterlegenheit der staatlichen gegenüber den privaten Ermittlern sobald wie möglich durch den deutschen Gesetzgeber wieder beendet werden muss. Wastl (Zwischenruf - Privatisierung staatsanwaltschaftlicher Ermittlungen, ZRP 2011, 57) sieht dies auch deshalb geboten, weil ,zwischenzeitlich auch in der US-amerikanischen Rechtsprechung der allzu simple Trick der Verlagerung staatsanwaltschaftlicher Ermittlungen auf Private als das enttarnt wurde, was es nun einmal ist: Der Versuch einer Umgehung rechtsstaatlicher Garantien!“. Sobald die wirtschaftlichen Schwerpunktstaatsanwaltschaften personell und ausrüstungsmäßig nachgerüstet haben, gibt es auch für die Unternehmen keine Veranlassung mehr, zur Besänftigung ausländischer Behörden ( $S E C$ und $D o J$ ) auf ihre Kosten teure Privatermittler zur personellen und finanziellen Entlastung staatlicher Behörden ins Unternehmen zu holen, die naturgemäß die Mitarbeiter nicht nur verunsichern, sondern manchmal auch von ihrer eigentlichen Arbeit abhalten.

\section{Privatization of criminal prosecution — a royal road or a wrong track?}

\section{Summary}

The comprehensive use of private individuals by the company management for the systematic clearance of suspected economic crimes has been for decades a tried and tested fact-finding method in the US legal system. In Germany this form of corporate private investigations took not 
place or was unknown to the public prosecution. This changed dramatically with the discovery of spectacular corruption affairs with international relation in 2007. "Internal Investigations" have developed since then to a lucrative commercial model particularly for American solicitor's offices, because only these are accepted by the American authorities (America only).

However, the adherence to examination standards of a state under the rule of law must be also protected in the job. The judiciary must win back the inquiry sovereignty in the fight against economic crime.

The fight against international economic crime can no longer be left to a single country alone. The fight against anti-competitive corruption should be a priority for the entire community. As an adequate means supplements to the Convention on the Fight against Corruption of the Council of the Organization for Economic Cooperation and Development (OECD) on the Laws of Transnational Criminal Prosecution of Economic Offenses come into consideration.

Keywords: privatization of criminal proceeding, criminal prosecution, private investigations, economic crimes, corruption, Transnational Criminal Prosecution. 